\title{
Performance study for the CEPC ScW ECAL
}

\section{Zhigang Wang ${ }^{1}$, Hang Zhao, Tao Hu}

State Key Laboratory of Particle Detection and Electronics(Institute of High Energy Physics, CAS)

19B Yuquan Road, Beijing, China

E-mail: wangzhg@ihep.ac.cn, zhaohang@ihep.ac.cn, hut@ihep.ac.cn

Electromagnetic calorimeter of the CEPC detector is employed for precise energy measurement of electron, photon, tau and hadronic jets. The scintillator-tungsten based electromagnetic calorimeter (ScW ECAL) is a sampling calorimeter consists of absorber and sensitive layers. In the baseline design the ScW ECAL is scintillator strip coupled with a SiPM. The performance of the ScW ECAL has been studied in PFA package .The light output of the scintillator strip with different size has been studied. The performance of SiPMs has been investigated and compared in our laboratory. 


\section{Introduction}

Electromagnetic calorimeter of the CEPC detector is employed for precise energy measurement of electron,photon,tau and hadronic jets. To fully exploit the physics potential about Higgs,W,Z and related SM processes[1], the jet energy resolution $\sigma_{\mathrm{E}} / \mathrm{E}$ is required to reach $3 \%-4 \%$, or $30 \% / \sqrt{E}$ at energies below about $100 \mathrm{GeV}$. This resolution is about a factor of two smaller than the calorimeters used for the LEP detectors and currently operating calorimeters at the LHC. The Particle Flow Algorithm[2](PFA) is a promising way to achieve the required jet energy resolution. The particle flow paradigm has tremendous impact on the design of the electromagnetic calorimeter detector. A scintillator-tungsten sandwich sampling calorimeter(ScW ECAL) is proposed to build a fine-segmented calorimeter in a stable,robust and cost effective way. The design of ScW ECAL is based on the ILD detector conpects[3].

\section{Structure of the ScW ECAL}

The proposed structure of the ScW ECAL is shown in Figure 1. The ScW ECAL consists of a cylindrical barrel system and two large end caps. In order to get an adequate energy resolution, the ScW ECAL is longitudinally segmented into around 25 layers, and the tungsten plate may have different thicknesses to reduce the number of readout channels. The active layers are plastic scintillators consisting of $5 \times 45 \mathrm{~mm}^{2}$ scintillator strips. The scintillator strips in adjacent layers are perpendicular to each other to achieve a $5 \times 5 \mathrm{~mm}^{2}$ effective transverse size. Photons from each scintillator strip are read out by compact photon sensro, SiPM, attached at the end of the strip. Thanks to the strip structure, number of readout channels can be significantly reduced. Keeping the required granularity and these merits, the ScW ECAL has good energy resolution and linearity. A super-layer is made of a tungsten plate $(3 \mathrm{~mm}$ thick), scintillator strips( $2 \mathrm{~mm}$ thick), and a readout/service layer( $2 \mathrm{~mm})$ thick .The total ScW ECAL thickness is $175 \mathrm{~mm}$, and $21.4 \mathrm{X}_{0}$ in radiation length.

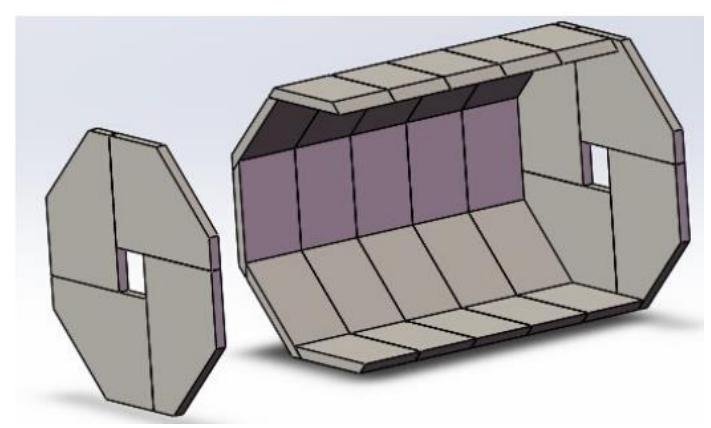

Figure 1. Structure of the ScW ECAL

\section{Carlo simulation study}

The performance of theScW ECAL has been studied in the simulation software Mokka[4]. In the Monte Carlo program, there are only plastic scintillator and tungsten plate, no readout layer include. The plastic scintillator is covered with $0.1 \mathrm{~mm}$ reflector film. The depedence of energy resolution of ECAL on thichkess of scintillator has been studied. As shown in Fig.2 left, with 
the thickness of the plastic scintillatorstrip changed from $3 \mathrm{~mm}$ to $1 \mathrm{~mm}$, the energy resolution is slightly worse. The relationship between energy resolution and number of layers has been studied, as shown in Fig.2 right. The layer number of CEPC ScW ECAL will be optimized between 20 to 25 .
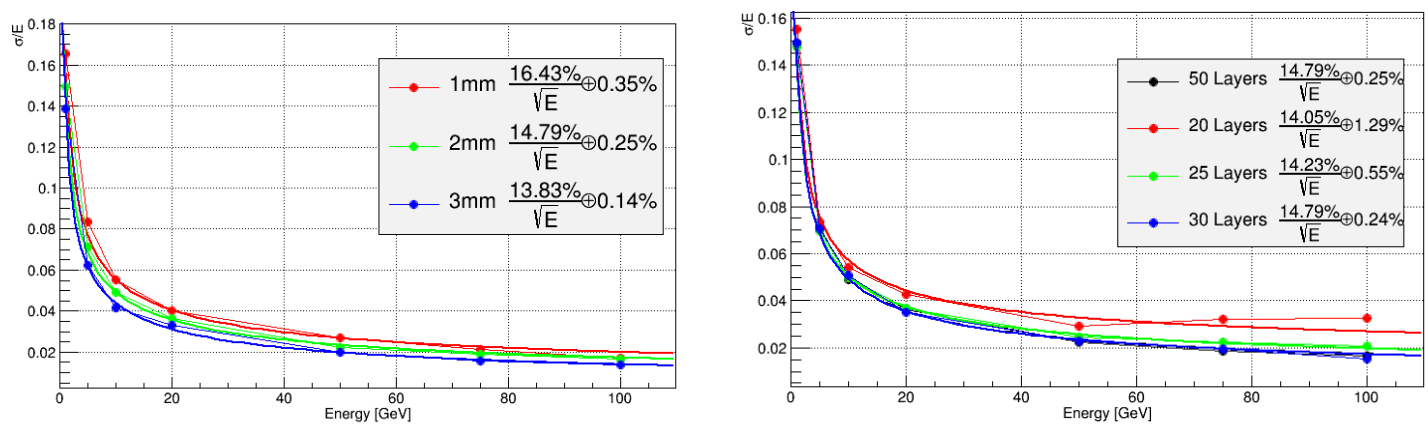

Figure 2 Depedence of energy resolution on thickness of scintillator(left)and layer number(right)

\section{SiPM performance study}

The performance of different SiPMs has been investigated in our laboratory. The SiPM is a solid-state device so it generates noise due to thermal excitation. The noise component is amplified in Geiger mode operation and the original photon detection signal cannot be discriminated from the noise. The noise occurs randomly so its frequency is a crucial parameter in determining SiPMs device characteristics. SiPMs with trenches between pixels dramatically reduced dark rate and pixel to pixel cross-talk. The dependence of dark count rate and crosstalk rate on operating voltage are shown in Fig.3. The performance of the latest devices has been improved greatly. This feature is very favorable for CEPC ECAL project. The SiPMs dynamic range is determined by the number of pixels. The manufactures have developed the SiPM with the pixel pitch of 10um, which increase the number of pixel per unit area, drastically extends the SiPMs dynamic range. But the photon detection efficiency of $10 \mathrm{um} \mathrm{SiPMs}$ is only $1 / 3$ of $25 \mathrm{um}$ SiPMs(from vendor's data sheet).
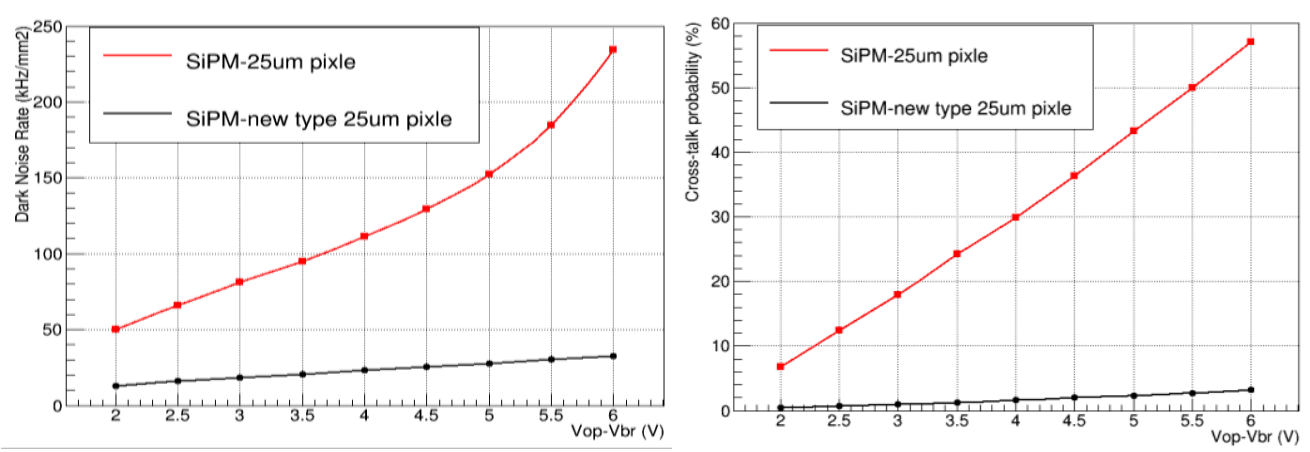

Figure3 The dependence of dark count rate (left) and crosstalk rate (right) on operating voltage

\section{Scintillator strip test}

The scintillator strip is thev sensitive material of the CEPC ScW ECAL. In the baseline design, the SiPM is attached to the end of the scintillator strip directly. The scintillator strip response has been test by radioactive source $\mathrm{Sr} 90$. Several scintillator strips with different shape are prepared and tested in our laboratory. Test results of light output of scintillator strip with 
dimensions of $5 \mathrm{~mm} \times 2 \mathrm{~mm} \times 45 \mathrm{~mm}$ and $10 \mathrm{~mm} \times 2 \mathrm{~mm} \times 90 \mathrm{~mm}$ are shown in Fig.4. The light output of the strip close to the SiPM is much larger than other position. The optimization of scintillator strip need much work to do in future. For CEPC ECAL the SiPM with 10000 pixel number is minimum requirements. The light output of strip with 10000 pixel SiPM is tested. It can be calculated that the photon detection efficiency of 10000 pixel SiPM is only $23 \%$ of the 1600 pixel ones.
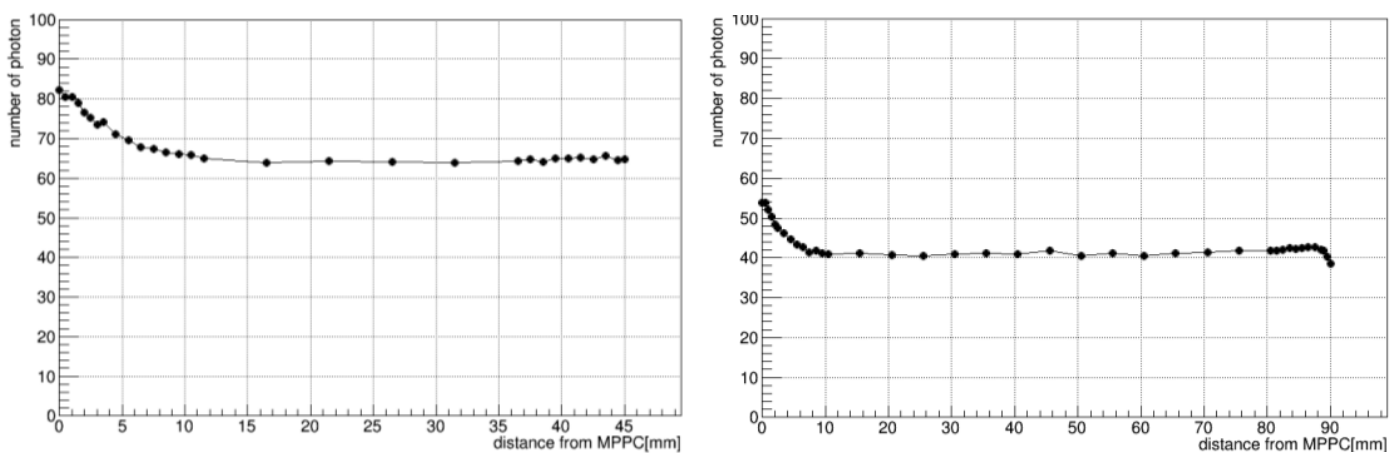

Figure 4 Light output of the scintillator strip with size of $5 \mathrm{~mm} \times 2 \mathrm{~mm} \times 45 \mathrm{~mm}$ (left) and $10 \mathrm{~mm} \times 2 \mathrm{~mm} \times 90 \mathrm{~mm}$ (right)

\section{Summary}

The CEPC ScW ECAl is a scintillator-tungsten sandwich sampling calorimeter, which is proposed to build a fine-segmented calorimeter in a stable,robust and cost effective way. The performance of readout unit of CEPC ScW ECAl has been tested. The parameters of SiPMs such as noise rate, cross-talk and dynamic range had been studied. The light output unifromaity of scintillator trip need to be improved in future.

\section{References}

[1] CEPC-SPPC Preliminary Conceptual Design Report Volume I-Physics\& Detector, the CEPC-SPPC Study Group

[2] M.Thomson, Particle Flow Calorimetry and the PandoraPFA Algorithm,Nucl. Instrum. Meth. A611 (2009)25-40, arXiv: 0907.3577[physics.ins-det].

[3] ILC Project, A. Djouadi et al.,eds.,International Linear Collider Reference Design Report Volume 2: Physics at the ILC. 2007.

[4] P.Mora de Fretas and H. Videau, Detectorsimulation with MOKKA/GANT4:Present and future, pp.623-627.20002.LC-TOOL-2003-010. 